\title{
Age changes of antyoxidant system and free-radical processes in rats at formation of postinfarction cardiosclerosis
}

\author{
Tatiana Rebrova*, Sergey Afanasiev, Olesia Putrova, Roman Batalov, Sergey Popov \\ Federal State Budgetary Institution, "Research Institute for Cardiology" of Siberian Branch, The Russian Academy of Medical \\ Sciences, Tomsk, Russia; ${ }^{*}$ Corresponding Author: rebrova@cardio.tsu.ru
}

Received 17 October 2012; revised 18 November 2012; accepted 26 November 2012

\section{ABSTRACT}

Estimation of age dependent changes of the lipid peroxidation (LPO) intensity, content of stable metabolites of nitrogen oxides (NO) and antioxidant enzyme activities in rat blood serum in conditions of experimental postinfarction cardiosclerosis (PICS) is carried out. Initiation of the postinfarction remodeling of animals has been carried out with coronary occlusion, definition of LPO and NO metabolites indices has been performed after 45 days. Investigations have been carried out on 44 and twelvemonth-old male Wistar rats with mass $200-250$ $\mathrm{g}$ and $400-450 \mathrm{~g}$, accordingly. Statistical analysis of the results was performed using the Mann-Whitney-Wilcoxone criterion. It has been found that already intact animals have age specificity of indices under consideration. The expressed activity of LPO processes on the background of reduction of endogenous fermentative antioxidant (SOD and catalase) activity as well as nitrite concentration in blood serum is characteristic for PICS of 4-month-old animals. PICS of 12-month-old rats is accompanied with suppression of the LPO processes on the background of reduction of the antioxidant enzyme intensity and increase of NO metabolites production. The following conclusions have been drawn. Process of ontogenesis is characterized by imbalance between pro- and antioxidant processes in rat blood. Increase in catalase activity and concentration of the TBC-active products at simultaneous decrease of SOD activity and content of diene conjugates has been noted. The organism of young animals responds with persistent increase of LPO processes and decrease of SOD and catalase activity on formation of postinfartion cardioslerosis. The less expressed increase of lipid peroxidation activation and de- crease of catalase activity has been noted in the organism of old animals within $\mathbf{4 5}$ days after PICS formation.

Keywords: Lipid Peroxidation; Nitrogen Oxide; Postinfarction Cardiosclerosis; Age-Related Aspect

\section{INTRODUCTION}

Free radical reaction has a definite place in the processes that occur in the alive organisms. The main source of radicals in organism is oxygen. Formation of free oxygen radical can occur at transfer of electrons in respiratory chain of mitochondrions [1,2], in NADP-cytochrome of P450-dependent system of microsomal oxidation of xenobiotics [3], at metabolism of purines and pyrimidines on xantine oxidase way [4] etc. The many works devoting to regulatory role of free radicals have been published in the last years [5-7]. Influence of free radicals and products of peroxidation on sensitivity of cellular membranes to mediators [5], as well as on reflex mechanisms of regulation [8] has been shown. Change of lipid peroxidation intensity in aging process of organism has been found $[5,9,10]$. The free radicals are very aggressive molecules which enter easily in interaction with the main cellular components causing oxidative modification of lipids, thiol groups of proteins and nucleic acids [6]. Oxidative modification of molecules leading to structural disturbance in the membrane bilayer and decrease of functional activity of integral membrane proteins is the pathogenetic link of the many pathological processes $[6,11]$. Study of ischemia-reperfusion lesions of cardiac muscle is pressing question in cardiology. It has been shown in experiment that development of coronary occlusion infarction creates conditions for uncontrollable development of the peroxide processes as result of decrease in antioxidant enzymes activity [12]. At the same time the clear idea about intensity of processes of lipid peroxidation (LPO) and their pathogenetic role 
in development of postinfarction cardiac remodeling is absent. At that there is a high probability that changes occurring in the system of pro- and antioxidants at myocardial remodeling in young and aging organisms can differ.

The aim of the present study is investigation of the age dependent feature of change of LOP process intensity, content of stable metabolites of nitrogen oxide and antioxidant enzymes in rat blood serum at postinfarction cardiac remodeling.

\section{METHODS}

\subsection{Animal Groups and Experimental Model}

Investigations have been carried out on 40 male Wistar rats of 2 age groups. The four-month-old and twelvemonth-old animals were obtained from the vivarium of the Institute of Cytology and Genetics of the Siberian Branch of the Russian Academy of Sciences (Novosibirsk, Russia). During of the whole experiment the animals were kept under standard conditions on routine food and water intake.

The first group contained 10 experimental and 10 control four-month-old animals with mass $200-250 \mathrm{~g}$. The second group contained the same number of the experimental and control twelve-month-old rats with mass 400 $450 \mathrm{~g}$ accordingly. Myocardial infarction has been simulated in the experimental animals of all groups by means of coronary occlusion in the field of the high third of link coronary artery [13]. Post-infarction cardiosclerosis (PICS) has been formed in experimental animals within 45 days after coronary occlusion [14]. The animals which have been suffered false operation without coronary occlusion served in our investigation as control. Gravimetric control for change of the left ventricle has been performed in the all group of animals within 45 days. The estimation of myocardial lesion volume has been performed according to the method [15].

\subsection{Serum Samples}

Blood sampling has been performed in cooled test glass, blood samples has been centrifuged at $3000 \mathrm{rpm}$ during 10 minutes. Obtained serum was dispensed for aliquots and stored in liquid nitrogen until investigation moment.

\subsection{Methods of Estimation of LPO}

LPO activity in blood serum was estimated in reaction with 2-thiobarbituric acid (TBA) by measuring concentration of TBA-active products (TBAAP) [16]. The concentration of primary products of LPO - dien conjugates (DC) was measured in the hexane extracts of serum samples with spectrophotometer at $232 \mathrm{~nm}$ [17].

\subsection{Measurement of Catalase Activity in Serum Samples}

The activity of catalase was determined by the spectrophotometric method [18], based on the ability of hydrogen peroxide to form a stable stained complex with molybdenum salts.

\subsection{Measurement of SOD Activity in Serum Samples}

Superoxide dismutase (SOD) activity in samples was evaluated by method of spectrophotometric measurement of adrenochrome concentration increase at $480 \mathrm{~nm}$ [19]. Adrenochrome is formed at the autoxidation of adrenalin in alkaline medium which proceeds with formation of oxygen superoxide anion. SOD dismuting superoxide anion inhibits autoxidation process.

Accomplishing the biochemical methods for the determination of LPO products and antioxidative ferment (catalase and SOD) activity one used Sigma reagents.

\subsection{Method of Estimation of NO Metabolism in Blood Serum}

Content of stabile metabolites of nitrogen oxide (NO) - nitrates and nitrites - is defined by the Griss reaction with use of commercial reagents kit Nitric Oxide $\left(\mathrm{NO}_{2}^{-} / \mathrm{NO}_{3}^{-}\right)$Assay Kit (Assay Designs).

\subsection{Statistical Analysis}

Confidence of differences of obtained data was evaluated by use of Mann-Whitney range criterion and Wilcoxone criterion. Differences are considered statistically significant at $\mathrm{p}<0.05$.

\section{RESULTS}

It has been found that statistically significant increase of TBAAP on $25 \%$ and decrease of DC content on $20 \%$ is characteristic for the control twelve-month-old animals in comparison with the young animal group (Table 1). These age changes of LOP products indices appeared interrelated with the antioxidant enzyme system activity. So, the activity of SOD enzyme and catalase in major age group differed from values obtained for juveniles. It has been revealed statistically significant decrease of SOD activity in 5 times and opposite, increase of catalase activity more than 2 times. At definition of nitrites and nitrates it has been shown that values of this metabolites of the twelve-month-old animals of the control group were statistically significantly lower (Table 2 ) in comparison with the four-month-old animal group. The revealed age dependent differences in TBAAP and DC concentration and SOD and catalase activity can be reflected on change of these indices at development of 
Table 1. Indices of lipid peroxidation and antioxidant enzyme activity in blood serum of 4-month-old and 12-month-old rats (M \pm $\mathrm{m})$.

\begin{tabular}{ccccc}
\hline \multirow{2}{*}{ Group } & \multicolumn{3}{c}{ Index } \\
\cline { 2 - 5 } & $\mathrm{DC}, \Delta \mathrm{E}_{232} / \mathrm{ml}$ & TBAAP, $\mu$ mol/ $/$ & Catalase, $\mu$ catal/1 & SOD, $\mathrm{mol} / \mathrm{min} \cdot \mathrm{L}$ \\
\hline $\begin{array}{c}\text { Control animals, 4-month-old } \\
(\mathrm{n}=10)\end{array}$ & $1.03+0.08$ & $20.99+2.03$ & $20.52+1.63$ & $0.86+0.05$ \\
Animals with PICS 4-month-old & $1.96+0.09$ & $25.58+2.26$ & $15.92+0.95$ & $0.09+0.01$ \\
$(\mathrm{n}=10)$ & $\mathrm{p}_{1}<0.05$ & $\mathrm{p}_{1}<0.01$ & $\mathrm{p}_{1}<0.01$ & $\mathrm{p}_{1}<0.01$ \\
Control animals 12-month-old & $0.82 \pm 0.11$ & $25.32 \pm 5.44$ & $41.3 \pm 2.95$ & $0.153 \pm 0.039$ \\
$(\mathrm{n}=10)$ & $\mathrm{p}_{2}<0.05$ & $\mathrm{p}_{2}<0.05$ & $\mathrm{p}_{2}<0.001$ & $\mathrm{p}_{2}<0.05$ \\
Animals with PICS 12-month-old & $0.85 \pm 0.19$, & $12.53 \pm 1.92$ & $26.7 \pm 3.33$ & $0.087 \pm 0.031$ \\
$(\mathrm{n}=10)$ & $\mathrm{p}_{1}>0.05, \mathrm{p}_{2}<0.05$ & $\mathrm{p}_{1}<0.05, \mathrm{p}_{2}<0.05$ & $\mathrm{p}_{1}<0.05, \mathrm{p}_{2}<0.05$ & $\mathrm{p}_{1}<0.05, \mathrm{p}_{2}<0.001$ \\
\hline
\end{tabular}

Note: $\mathrm{p}_{1}$ - significant differences from that of the group of control animals of corresponding age; $\mathrm{p}_{2}$ - significant differences in a similar group of young animals; $\mathrm{n}$ - number of animals in group.

Table 2. Change of concentration of nitrites and nitrates in blood serum of the rats of various age groups at formation of PICS (M \pm $\mathrm{m})$.

\begin{tabular}{|c|c|c|c|}
\hline Anima & Index & Nitrites $\mu \mathrm{mol} / \mathrm{l}$ & Nitrates $\mu \mathrm{mol} / \mathrm{l}$ \\
\hline \multirow{2}{*}{ 4-month-old } & Control animals $(\mathrm{n}=10)$ & $2.655 \pm 0.598$ & $29.598 \pm 9.958$ \\
\hline & Animals with PICS $(n=10)$ & $1.499 \pm 0.342^{*}$ & $28.194 \pm 8.119$ \\
\hline \multirow{2}{*}{ 12-month-old } & Control animals $(\mathrm{n}=10)$ & $1.12 \pm 0.516^{\#}$ & $18.42 \pm 3.86^{\#}$ \\
\hline & Animals with PICS $(n=10)$ & $2.44 \pm 0.085^{* \#}$ & $28.85 \pm 6726^{*}$ \\
\hline
\end{tabular}

Note: ${ }^{*}(\mathrm{p}<0.05)$ - significant differences from indices in the group of control animals of corresponding age; ${ }^{\#}(\mathrm{p}<0.05)$ - significance differences of values in animals of older age group from the corresponding values in the 4 -month-old rats. $n$-number of animals in group.

postinfarction cardiosclerosis.

Average value of heart mass in the four-month-old animal group within 45 days after modeling of infarction exceeded similar index of the control rats on $22.8 \% \pm$ $3.11 \%(\mathrm{p}<0.01)$. At that the zone of postinfarction scar made in average $55.0 \% \pm 9.00 \%$ of the left ventricle mass. The average value of heart mass in the twelvemonth-old animal group increased on $28.9 \% \pm 2.58 \%(\mathrm{p}<$ 0.05 ) in comparison with control group of this age as result of postinfarction remodeling. Percentage of scarce tissue in the left ventricle made $58.0 \% \pm 9.90 \%$. Revealed changes testify that postinfarction cardiac remodeling leads to development of myocardial hypertrophy both of the young animals and the animals of major age group.

Results allowing to judge about LPO activity and stress of the endogenous antioxidant system at remodeling of myocardium are presented in Table $\mathbf{1}$. The average value of TBAAP contents in blood serum samples of the fourmonth-old animals at PICS exceeds indices obtained in the control animal group of the same age on more than $21 \%$. The more expressed differences were revealed by DC content. Its concentration in experimental animals exceeds values obtained in the control group on $90 \%$. At investigation of endogenous antioxidant enzyme activity we revealed that catalase and SOD activities in serum of the postinfarction four-month-old animals is less on $22 \%$ and $89 \%$ than in the control group of the same age, accordingly (Table 1). Definition of the concentration of stable NO metabolites-nitrites and nitrates-showed that myocardial postinfarction remodeling is accompanied with authentic decrease of nitrites (on 44\%) while the content of nitrates remains on the same level that in the control group (Table 2).

Similar investigations carried out on the twelve-monthold animals gave some other results. It has been found that on the background of developed postinfarction remodeling the average content of TBAAP in the rats of this age group are more than $50 \%$ less than one of the control animals of the same age while the content of DC practically has not been changed (Table 1). The significant $(\mathrm{p}<0.05)$ decrease of the SOD (on $43 \%)$ and catalase (on 35\%) activity in relation to indices of the control animal group was characteristic. Definition of stable NO metabolites (Table 2) showed increase $(p<0.05)$ of concentration of both nitrites (on 117\%) and nitrates (on $56 \%)$.

\section{DISCUSSION}

Activation of free-radical oxidation is considered as 
one of the aging mechanism of organism $[9,10]$. There is interconnection between aging of an organism and development of many pathologies including heart failure of ischemic genesis.

It is shown that at acute and chronic myocardial ischemia the production of active form of oxygen and activation of lipid peroxidation increases significantly and antioxidant potential decreases $[6,11,12]$. During whole postinfarction period myocardium suffers the complex of structural and functional changes which are noted not only in the lesion focus but and in the zones remote from lesion [20].

Excessive activation of the processes of free radical oxidation of lipids is one of the factors increasing permeability of cellular membranes as well as leading to oxidative modification of their structural proteins and enzymes [5,12].

Our investigation of free radical processes in the control animals of different age testify that aging process leads to increase of TBAAP concentration in blood serum. Such an increase can be considered as result of strengthening of their formation in animals of major age group or as result of decrease of utilization. However, we did not observe significant changes in DC content. It is known that intensity of metabolic processes in organism decreases and mitochondrial respiratory activity are depressed with age. Some authors connect decrease of number of active oxygen form molecules just with this fact $[21,22]$. Our investigation found that accumulation of LOP products in animals of major age group can be result of age dependent decrease of SOD activity [23]. Since we observed rise only TBAAP index which formation occurs in process of synthesis of thromboxane [24], it is possible to suppose that there is no real rise of free radical processes activity in the 12-month-old rats.

Behavior of LOP in living organisms is regulated by multicomponent antioxidant system which constraints excessive formation of hydroperoxides and lipid radicals $[1,5,6]$. SOD and catalase are high specific regulatory enzymes which cause antioxidant action and neutralize such an active forms of oxygen as superoxide anion radical and hydrogen peroxide $[1,5,6]$.

We revealed oppositely directed changes of SOD and catalasa activity in 4-month-old and 12-month-old animals. Decrease of SOD activity of the rats in major age group can be considered as evidence of suppression of antioxidative ability of organism. The most probably decrease of SOD activity is caused by inhibition of active center of this enzyme with LOP products [25]. Increase of catalase activity in the rats of major age group probably reflects realization of compensatory possibilities of endogenous antioxidant system.

Obtained results are corresponded with data of other researchers showing that SOD activity decreases in blood of the animals in age range from 3 up to 30 months and catalase activity, opposite, increases $[23,26]$.

Simulation of PICS in the 4-month-old rats in our investigations was accompanying with increase of TBAAP and DC content in blood serum that testifies intensive passing of LOP processes even within 45 days after simulation of myocardial infarction. Increase of indices of lipid peroxidation in serum can be result of exit of LOP products in blood flow from damaged myocardium or generalization of oxidative stress on the level of whole organism. In this case active passing of LOP processes can occur in membranes of the form elements of blood, endothelium and vessel walls.

In the most cases action of endogenous antioxidant enzymes is inefficient at oxidative stress as result of inhibiting action of fat acid peroxides and active forms of oxygen on their active centers $[25,27]$. It may well be that increased formation of active forms of oxygen and lipid peroxides which are typical for ischemic action $[6,11]$ promotes decrease of antioxidant enzyme activity $[25,27]$ that in certain conditions leads to formation of the peculiar "closed" circle and formation of oxidative stress. Decrease of SOD and catalase activity in blood serum of young animals with cardiosclerosis revealed by us can be considered as additional confirmation that state of oxidative stress remains in these animals and within 45 days after simulation of myocardial infarction.

Changes of DC and TBAAP indices of the rats of major age group suffered coronary occlusion were differently directed. At these terms of postinfarction remodeling we noted decreased TBAAP content with no significant changes in DC concentration.

Comparison of obtained results allows to draw conclusion that active remodeling of myocardium remains at young rats within 45 days after PICS which is expressed in particularly in intensive passing of LOP processes. Active passing of LOP processes was not observed in the old rats within 45 days after coronary occlusion. It can be considered that decrease of LOP intensity at cardiosclerosis in the old rats is achieved with the significant exhaustion of reserves of endogenous antioxidant system. It is corresponded to decrease of SOD and catalase activity in the old rat group revealed by us.

$\mathrm{NO}$ and its derivatives together with active forms of oxygen and lipid peroxides play significant role in pathological processes developing at myocardial ischemia. Variety of NO regulatory effects can promote demonstration both cytoprotective and cytotoxic action in various pathophysiological processes.

Acting through activation of guanilatcyclase and decrease of intracellular calcium, NO leads to relaxation of smooth muscle vessel tissue and improvement of blood flow of ischemic section that decreases grade of myocardial ischemic injury [28]. It is shown that NO cannot 
inhibit complexes of respiratory chain of mitochondria that is accompanied with decrease of macroenergetic phosphates in cells $[29,30]$. Interaction of NO with superoxide anion radical of oxygen leads to formation of toxical peroxynitrite which is strong cytotoxic agent and is able to induce LOP processes, to cause one-string breaks of DNA, and to nitrate aromatic amino acids [31]. Molecule of NO in physiological concentrations has antioxidant properties effectively binding with organic radicals of lipids to lead to break of chain reactions of freeradical oxidation [32]. Regulatory action of NO on SOD [33] and catalase [34] activity in conditions of ischemiareperfusion is shown. In our experiments we evaluated only integral effect of NO since we defined concentration of its stable metabolites and LPO products in blood serum but not in particular organs.

Decrease of nitrite and nitrate concentration in serum samples of the control animals of major age group testifies decrease of NO synthesis that can act as one of causes of age disregulation of lipid peroxidation processes and antioxidant enzyme activity. It is shown that NO causes inhibitive effect on catalase activity by means of binding with gemm of active centre of enzyme [34]. On this basis one can assume that increase of this enzyme activity with age occurs as result of decrease of concentration of inhibitive factor in organism of the rats of major age group. Previously, it was shown on the model of separated rat heart that preliminary insertion of blocator of synthesis NO-L-NAME prevented decrease of catalase activity caused by LOP induction [35]. Although SOD also is gem containing enzyme similar effect of NO blockade has not been noted.

Our investigation found that formation of PICS in young rats was accompanied with decrease of nitrite concentration and did not influence on nitrate level. Noted decrease of NO concentration, probably, is caused by its fast consumption in metabolite reactions. Development of PICS in the old rat groups was accompanied with increase of NO production up to level of young rats in control group. Probably, increase of NO concentration could promote decrease of catalase activity.

Thus, obtained results showed that in PICS conditions one observes in young rats expressed activity of LOP processes on the background of authentic decrease of endogenous enzyme antioxidants and NO concentration in blood serum. Myocardial remodeling of the old rats at PICS is accompanied with suppression of LOP processes on the background of the decrease of antioxidant enzyme activity and the increase of NO production.

\section{CONCLUSIONS}

1) Process of ontogenesis is accompanied with imbalance between pro- and antioxidant processes occurring in the blood of the laboratory rat. One notes the increase of catalase activity and TBAAP concentration at a simultaneous decrease of SOD activity and DC content;

2) Young animal organism responds with stable increase of LOP processes intensity and decrease of SOD and catalase activity on formation of postinfarction cardiosclerosis;

3) The less expressed activation of lipid peroxides and the decreased catalase activity have been noted in organisms of the old animals on the 45 days of PICS formation.

\section{REFERENCES}

[1] Vladimirov, Yu.A. (2000) Free radicals in biological systems. Soros Educational Journal, 12, 13-19.

[2] Chen, Q., Moghaddas, S., Hoppel, C.L., et al. (2008) Ischemic defects in the electron transport chain increase the production of reactive oxygen species from isolated rat heart mitochondria. American Journal of Physiology, 294, 460-466. doi:10.1152/ajpcell.00211.2007

[3] Cave, A.C., Brewer, A.C., Narayanapanicker, A., et al. (2006) NADPH oxidases in cardiovascular health and disease. Antioxidants \& Redox Signaling, 8, 691-728. doi:10.1089/ars.2006.8.691

[4] Sato, E., Mokudai, T., Niwano, Y., et al. (2011) Kinetic analysis of reactive oxygen species generated by the in vitro reconstituted NADPH oxidase and xanthine oxidase systems. The Journal of Biochemistry, 150, 173-181. doi:10.1093/jb/mvr051

[5] Dröge, W. (2002) Free radicals in the physiological control of cell function. Physiological Reviews, 82, 47-95.

[6] Valko, M., Leibfritz, D., Moncol, J., et al. (2007) Free radicals and antioxidants in normal physiological functions and human disease. The International Journal of Biochemistry \& Cell Biology, 39, 44-84. doi:10.1016/j.biocel.2006.07.001

[7] Karageuzyan, K.G. (2005) Oxidative stress in the molecular mechanism of pathogenesis at different diseased states of organism in clinics and experiment. Current Drug Targets-Inflammation \& Allergy, 4, 85-98. doi:10.2174/1568010053622939

[8] Prabhakar, N.R. (2011) Sensory plasticity of the carotid body: Role of reactive oxygen species and physiological significance. Respiratory Physiology \& Neurobiology, 178, 375-380. doi:10.1016/j.resp.2011.05.012

[9] Anisimov, V.N. (2008) Molecular and physiological mechanisms of aging. Publishing House Nauka, St. Petersburg.

[10] Spiteller, G. (2007) The important role of lipid peroxidation processes in aging and age dependent diseases. Molecular Biotechnology, 37, 5-12. doi:10.1007/s12033-007-0057-6

[11] Mirsa, M.K., Sarwat, M., Bhakuni, P., et al. (2009) Oxidative stress and ischemic myocardial syndromes. Medical Science Monitor, 15, 209-219.

[12] Lankin, V.Z., Tikhaze, A.K. and Belenkov, Yu.N. (2000) Free radical processes in diseases of the cardiovascular system. Kardiologiya, 7, 48-61.

[13] Gomez, A.M., Guatimosim, S., Dilly, K.W., et al. (2001) 
Heart failure after myocardial infarction: Altered excitation-contraction coupling. Circulation, 104, 688-693. doi:10.1161/hc3201.092285

[14] Rebrova, T.Yu., Kondratieva, D.S. and Afanasiev, S.A. (2007) Activity of lipid peroxidatin and functional state of the myocardium in remodeling of rat heart after experimental myocardial infarction. Kardiologiya, 6, 41-45.

[15] Schultz, J.E.J., Hsu, A.K., Nagase, H., et al. (1998) TAN67 , a $\delta 1$-opioid receptor agonist, reduces infarct size via activation of $\mathrm{G}_{\mathrm{i} / \mathrm{o}}$ proteins and KATP channels. American Journal of Physiology, 274, H909-H914.

[16] Korobeinikova, E.N. (1989) Modification of the definition of lipid peroxidation products in the reaction with thiobarbituric acid. Laboratory Work, 7, 8-10.

[17] Bolland, J.L. and Koch, H.P. (1945) The course of antioxidant reaction in polyisoprenes and allied compounds. Part IX. The primary thermal oxidation product of ethyl linoleate. Journal of the Chemical Society, 7, 445. doi:10.1039/jr9450000445

[18] Karalyuk, M.A., Ivanov, L.I., Mayorov, I.G., et al. (1988) The method for determining the activity of catalase. Laboratory Work, 1, 16-19.

[19] Brusov, O.S., Gerasimov, A.M. and Panchenko, L.F. (1976) The influence of natural inhibitirs of free radical reaction on epinephrine autooxidation. Bulletin of Experimental Biology and Medicine, 1, 33-34.

[20] Slezak, J., Tribulova, N., Ravingerova T., et al. (1992) Myocardial heterogeneity and regional variations in response to injury. Laboratory Investigation, 67, 322-330.

[21] Barja, G. (1999) Mitochondrial oxygen radical generation and leak: Sites of production in states 4 and 3, organ specificity, and relation to aging and longevity. Journal of Bioenergetics and Biomembranes, 31, 347-366. doi:10.1023/A:1005427919188

[22] Sohal, R.S. and Weindrich, R. (1996) Oxidative stress, caloric restriction and aging. Science, 273, 59-63. doi:10.1126/science.273.5271.59

[23] Voskresenskii, O.N., Zhutaev, I.A., Bobyrev, V.N., et al. (1982) Antioxidant system ontogenesis and aging. Problems of Medical Khimii, 1, 14-27.

[24] Halliwell, B. (2000) Lipid peroxidation, antioxidants and cardiovascular disease: How should we move forward? Cardiovascular Research, 47, 410-418. doi:10.1016/S0008-6363(00)00097-3

[25] Lankin, V.Z., Vandyshev, D.B. and Tikhaze, A.K. (1981) Effect of hyperoxia on superoxide dismutase and gluta- thione peroxidase in the tissues of mice. Doklady of the Academy of Sciences of the USSR, 259, 229-231.

[26] Kogan, A.Kh., Kudrin, A.Kh., Kaktursky, L.V., et al. (1992) Peroxide free radical mechanisms of the pathogenesis of myocardial ischemia and enzyme regulation. Patophysiology, 2, 5-15.

[27] Gudz, T.I., Peshkova, E.G. and Goncharenko, E.N. (1982) Inhibition of superoxide dismutase activity by linolenic acid hydroperoxide. Radiobiology, 22, 674-677.

[28] Lundberg, J.O. and Weitzberg, E. (2005) NO generation from nitrite and its role in vascular control. Arteriosclerosis, Thrombosis, and Vascular Biology, 25, 915-922. doi:10.1161/01.ATV.0000161048.72004.c2

[29] Gutierrez, J., Ballinger, S.W., Darley-Usmar, V.M., et al. (2006) Free radicals, mitochondria, and oxidized lipids: The emerging role in signal transduction in vascular cells. Circulation Research, 99, 924-932. doi:10.1161/01.RES.0000248212.86638.e9

[30] Beltrán, B., Mathur, A., Duchen, M.R., et al. (2000) The effect of nitric oxide on cell respiration: A key to understanding its role in cell survival or death. Proceedings of the National Academy of Sciences of the United States of America, 97, 14602-14607. doi:10.1073/pnas.97.26.14602

[31] Zweier, J.L., Fertmann, J. and Wei, G. (2001) Nitric oxide and peroxynitrite in postischemic myocardium. Antioxidants \& Redox Signaling, 3, 11-22. doi:10.1089/152308601750100443

[32] O'Donnel, V.B. and Freeman, B.A. (2001) Interactions between nitric oxide and lipid oxidation pathways: Implications for vascular disease. Circulation Research, 88, 12-21. doi:10.1161/01.RES.88.1.12

[33] Yang, B.C. and Mehta, J.L. (1997) Inhibition of nitric oxide does not affect reperfusion-induced myocardial injury, but it prevents lipid peroxidation in the isolated rat heart. Life Sciences, 61, 229-236. doi:10.1016/S0024-3205(97)00378-0

[34] Brown, G.C. (1995) Reversible binding and inhibition of catalase by nitric oxide. European Journal of Biochemistry, 232, 188-191. doi:10.1111/j.1432-1033.1995.tb20798.x

[35] Rebrova, T.Yu., Maslov, L.N., Lishmanov, A.Yu. and Tam, S.V. (2001) Stimulation of $\mu$ - and $\delta$-opite receptors and tolerance of isolate heart to oxidative stress: The role of NO-syntase. Biochemistry (Moscow), 66, 422-428. doi:10.1023/A:1010253530026 